\title{
Editorial -Circuler, refouler, enfermer, éloigner : zones d'attente et centre de rétentions aux frontières des démocraties occidentales
}

Didier Bigo

\section{(2) OpenEdition \\ Journals}

Édition électronique

URL : http://journals.openedition.org/conflits/344

DOI : $10.4000 /$ conflits.344

ISSN : $1777-5345$

Éditeur :

CCLS - Centre d'études sur les conflits lilberté et sécurité, L'Harmattan

Édition imprimée

Date de publication : 15 octobre 1996

ISSN : 1157-996X

Référence électronique

Didier Bigo, «Editorial -Circuler, refouler, enfermer, éloigner : zones d'attente et centre de rétentions aux frontières des démocraties occidentales », Cultures \& Conflits [En ligne], 23 | automne 1996, mis en ligne le 15 mars 2006, consulté le 30 mars 2021. URL : http://journals.openedition.org/conflits/344 ; DOI : https://doi.org/10.4000/conflits.344

Ce document a été généré automatiquement le 30 mars 2021.

Creative Commons License 


\title{
Editorial -Circuler, refouler, enfermer, éloigner : zones d'attente et centre de rétentions aux frontières des démocraties occidentales
}

\author{
Didier Bigo
}

La France vient de connaître avec le débat autour des "sans papiers" une forte polémique. Pour le gouvernement la situation illégale de la plupart des personnes les empêche d'avoir un droit à exiger des droits. Ils peuvent tout au plus demander des conditions plus humaines pour leur retour ou un certain délai. Pour les associations et partis d'opposition mobilisés auprès des sans papiers, la situation illégale a été créée par le gouvernement et l'inconséquence des lois Pasqua de 1993. On ne pourrait donc leur reprocher leur illégalité qui n'est certainement pas, dans ce cas, une clandestinité sur le territoire français. Le collège des médiateurs avait cru pouvoir un moment convaincre le gouvernement d'une nécessité d'aller au-delà de l'application stricte de la loi, mais les événements ont montré le contraire. Nos collègues d'Esprit, d'Hommes et Migration, du Monde Diplomatique ont déjà rendu compte avec finesse des ambiguïtés des différentes positions, en particulier gouvernementale ${ }^{1}$. Le débat s'est centré sur l'application des lois Pasqua et leur éventuelle réforme. I1 a abordé le thème des politiques d'immigration en s'interrogeant sur les besoins de l'économie française et sur l'hypocrisie d'une « inclusion clandestine » ou d'une «back door » à la «forteresse française ». On a discuté des capacités ou non des Etats européens, ensemble ou séparément, à fermer leurs frontières aux flux de personnes transfrontières et à bloquer l'immigration clandestine. Mais on s'est peu interrogé sur les lieux d'enfermement (zones d'attente et centres de rétention ${ }^{2}$ ) où se concentrent sans doute les situations les plus délicates et on a eu tendance à croire à une exceptionnalité des lois Pasqua, qui, dès lors, pourraient être facilement réformées. Nous voudrions dans ce numéro essayer d'approfondir ces questions en combinant une approche comparée des 
modalités d'enfermement et de renvoi qui touchent les personnes en situation illégale et en ayant recours à une pluridisciplinarité nécessaire (approches de droit public, des droits de l'homme, historique, de science politique). Tout d'abord en donnant une dimension comparative des pratiques de renvoi, d'enfermement, d'expulsion enregistrées en France, au Royaume-Uni, en Allemagne, aux Etats-Unis. nous pourrons mieux prendre la mesure des contraintes auxquelles les gouvernements se disent confrontés et nous pourrons voir quelles ont été leurs stratégies. Ensuite en intégrant cette question spécifique des zones d'attente et des centres de rétention, c'est à dire des pratiques d'enfermement, au sein d'une réflexion plus globale sur les logiques mises en oeuvre pour ces renvois, nous espérons montrer les rationalités administratives qui tendent à deshumaniser les situations de détresse dans lesquelles se trouvent les individus "sans papiers». En effet il importe d'abord de connaitre les régimes juridiques et l'application des lois surtout lorsqu'elles diffèrent d'un pays à l'autre. Cela montre toute la force de la spécificité des trajectoires nationales, même si le contexte européen joue et a poussé les différentes législations nationales à aller vers toujours plus de rigueur, la libéralité étant assimilé à une faiblesse et à un trou dangereux dans la digue législative des frontières de l'espace européen. II faut aussi rendre compte des luttes menées au nom des droits de l'Homme et du registre que ces luttes introduisent. I1 est nécessaire de faire toucher du doigt la quotidienneté des conditions de vie dans ces centres de rétention et zone d'attente, tant de ceux qui y sont retenus que de ceux qui les surveillent. Il faut analyser les mécanismes de résistance et la différence de leurs points d'application. Il faut enfin resituer dans une approche théorique plus globale la logique de ces pratiques d'enfermement en essayant de répondre à la question: comment la circulation des personnes débouchent-elles sur des formes originales d'enfermement (zones d'attente et centres de rétention), qui en disent long sur nos sociétés? En effet, si comme le supposait Foucault, la prison en enfermant débouchait sur l'exil et si elle était la forme disciplinaire d'une certaine modernité, les centres de rétention ne sont-ils pas la forme correspondante d'une post-modernité qui vise moins à corriger et discipliner les individus qu'à accélérer les flux, qu'à les suivre et les anticiper, qu'à les maitriser ? ${ }^{3}$ Dans ce numéro, François Julien-Laferrière présente les mécanismes juridiques qui règlent les conditions des zones d'attente et des centres de rétention en France. Un dossier constitué des travaux de l'ANAFE, du GISTI, de l'ouvrage témoignage de Jean-Pierre Perrin Martin, des commissions parlementaires et de leurs auditions ainsi que d'entretiens réalisés pour Cultures \& Conflits par Hugo Salinas, donne une série d'éléments statistiques et apporte l'éclairage des acteurs en reprenant et en mettant en série leurs témoignages. Barbara Harrell-Bond et Enoch Opongo reviennent sur la situation anglaise en analysant comment les rhétoriques antiétrangers ont depuis longtemps été intégrées comme partie du jeu politique. Ils s'émeuvent des pratiques menées dans le pays de l'habeas corpus où la privatisation de certaines fonctions de sécurité comme celles du gardiennage concernant ces centres débouche sur des formes d'arbitraire et montrent que le Royaume-Uni est sans doute, par son absence de formalisme, celui où les situations concrètes des personnes retenues sont les plus difficiles. Frank-Paul Weber analyse quant à lui, sur la longue durée la manière dont citoyenneté étatique et définition des étrangers à travers les pratiques d'expulsion se configurent mutuellement et il propose une lecture de l'enfermement et des pratiques de résistance dans les centres de rétention en Allemagne qui éclaire sur les rationalités de ces pratiques. S'appuyant, comme nous l'avions suggéré lors de la table ronde de 1994, sur une grille de lecture foucaldienne, il met en évidence la 
corrélation entre les discours, les savoirs, les pratiques des multiples acteurs qui interviennent dans le dispositif enfermement-éloignement. Samuel Schmidt dégage quant à lui les grandes lignes de la politique américaine à l'égard des Mexicains à travers l'étude des mécanismes de renvoi et de maintien en détention. Il analyse de manière structurelle les raisons du départ des Mexicains vers les Etats-Unis et la coresponsabilité de ces derniers dans cet exode. Il montre qu'aucune solution policière ne pourra régler ce régime des inégalités économiques et politiques qui subsistent entre les deux pays et qui se voit de manière criante à la frontière. Notre prochain numéro développera lui les questions du passage des frontières, de l'asile, de l'immigration afin de resituer les dispositifs d'enfermement-éloignement dans les logiques qui les constituent. Didier Bigo, Albrecht Funk, Mike King, Anastasia Tsoukala pour l'Europe et Randy Willoughby pour les Etats-Unis reviendront sur les logiques d'inclusion et d'exclusion qui poussent d'une certaine manière à la création de ces lieux d'enfermement et de renvoi.

\section{NOTES}

1. Cf. Joël Roman, «Les sans papiers de Saint Bernard », Esprit, octobre 1996 ; Hommes et Migrations, octobre 1996 ; Le Monde Diplomatique, septembre 1996.

2. Les termes zones d'attente et centres de rétention ont été retenus pour parler des diverses situations mais les appellations juridiques diffèrent d'un pays à l'autre. Hors de France la distinction rétention détention n'a pas la même portée juridique.

3. Cette hypothèse sera développée dans notre prochain numéro.

\section{INDEX}

Mots-clés : centre de rétention, frontières, mobilité, contrôle, étrangers 